\title{
COLLABORATIVE OPTIMIZATION OF MANAGEMENT STRATEGY WITH ENERGY INTERACTION AND SIZING FOR THE TRAM'S HYBRID STORAGE SYSTEM
}

\author{
YU WANG, ZHONGPING YANG \& FEI LIN \\ School of Electrical Engineering, Beijing Jiaotong University, China
}

\begin{abstract}
Energy management strategy (EMS) and sizing are the key steps in the design of a tram's power system and the result has a direct influence on the operation characteristics and economic benefit. This paper proposed an improved EMS with energy interaction and made collaborative sizing optimization based on actual vehicle and line conditions. The superiority of the improved EMS was shown through the sizing result and was verified on the experiment platform at the same time. Simulation and experiment showed that the improved EMS reduced the weight of hybrid storage system and guaranteed the normal operation even at emergency.
\end{abstract}

Keywords: hybrid storage system, sizing, energy management strategy.

\section{INTRODUCTION}

As a kind of irreplaceable transportation, modern tram has the advantages of large passenger volume, environmental protection, energy saving and relatively low cost. It is suitable for popularization and application in medium-sized cities. In recent years, with the implementation of clean energy policy and the demand for safety and aesthetics, the tram with onboard storage system has been widely studied. To meet the needs of high power and high energy consumption in the operation process, the onboard storage system should be carefully designed. The energy density of the battery is high, but the power density is low. On the contrary, the power density of the supercapacitor is high, but the energy density is low. In order to improve the dynamic performance of tram, the hybrid energy storage system with battery and supercapacitor has become a hot research direction.

EMS determines the performance of the tram's hybrid energy storage system, and the appropriate EMS can not only make the tram running safely and smoothly, but also reduce the loss and the operation cost. EMS can be distributed into two types, online strategy and offline strategy. There are many types of online strategy such as the logic threshold approach [1]-[3], frequency filtering strategy [4]-[5] and the strategy based on fuzzy logic rules [6][7]. Their rules are relatively simple and easy to achieve, but they are not dynamic enough to be adjusted at any time, and the rules need to be formulated based on certain experience. However, the offline strategy such as dynamic programming strategy [8]-[9], although the global optimal solution can be obtained, there are more restrictions on the use and it is difficult to apply in the actual operation.

Sizing is the key step of the tram's hybrid storage system optimization, and it has an impact on the characteristics of the energy storage system. Sizing seems to only influence the weight and volume of the hybrid storage system, nevertheless, the initial cost, life-cycle cost, battery lifespan, vehicle efficiency, and other elements are relative to sizing optimization. The procedure can be simple if it only considered the boundary conditions such as energy consumption, peak power, weight limitation, and terminal voltage. The sizing optimization and EMS interacts and restricts each other.

The paper improved EMS based on fixed power ratio strategy, adding the energy interaction state when the supercapacitor lacks enough energy. And made collaborative 
sizing optimization based on the improved EMS and verified the superiority of the EMS through contrast experiment.

This paper is organized as follows. Section 2 provides the model of the hybrid storage system and the improved EMS. Section 3 introduces the detailed process of collaborative sizing optimization. A detailed case based on real condition of trams and lines as well as contrast experiment are shown in Section 4. And Section 5 presents the conclusions of the paper.

\section{ENERGY MANAGEMENT STRATEGY}

The topology of hybrid storage system used in tram is shown in Fig. 1. The system is distributed into battery pack and supercapacitor pack, they discharge and charge according to the control signal to provide traction power and auxiliary power and to absorb regeneration power.

The model of battery is the "Rint" battery model [10], [11], as shown in Fig. $1, \mathrm{R}_{\mathrm{b}}$ is the internal resistances of the battery pack. The model of supercapacitor is the "RC" capacitor model, and the resistance Rsc is shown in Fig. 1.

The equations concerning power loss $P_{\text {loss }}$, operation efficiency $\eta_{\mathrm{HEV}}$ and energy loss $\mathrm{E}_{\text {loss }}$ are as follows:

$$
\begin{aligned}
& P_{\text {loss }}(k)=\left\{\begin{array}{l}
\mathrm{P}_{\text {req }}(k) \cdot\left(\frac{1}{\eta_{d c}}-1\right)+P_{r l o s s}(k), P_{r e q}(k) \geq 0 \\
\mathrm{P}_{\text {req }}(k) \cdot\left(\eta_{d c}-1\right)+P_{r l o s s}(k), P_{r e q}(k)<0
\end{array}\right. \\
& P_{r l o s s}(k)=I_{b}^{2}(k) \cdot R_{b}+I_{s c}^{2}(k) \cdot R_{s c} \\
& \eta_{H E V}(k)=1-\frac{P_{\text {loss }}(k)}{\mathrm{P}_{r e q}(k)}+P_{\text {loss }}(k) \\
& E_{\text {loss }}(k)=\sum P_{\text {loss }}(k) \cdot \Delta t(k) \\
& k=1,2, \ldots, N
\end{aligned}
$$

where $\mathrm{P}_{\text {req }}$ is the required power from the DC bus; $\mathrm{P}_{\mathrm{b}}$ and $\mathrm{P}_{\mathrm{sc}}$ are the power requirements of the battery pack and supercapacitor pack, respectively; $\eta_{d c}$ is the efficiency of every DC/DC converter; $\mathrm{I}_{\mathrm{b}}$, and $\mathrm{I}_{\mathrm{sc}}$ are the currents of battery pack and supercapacitor pack, respectively; and $\Delta \mathrm{t}$ is the time interval. To protect the energy storage system and prevent excessive power,

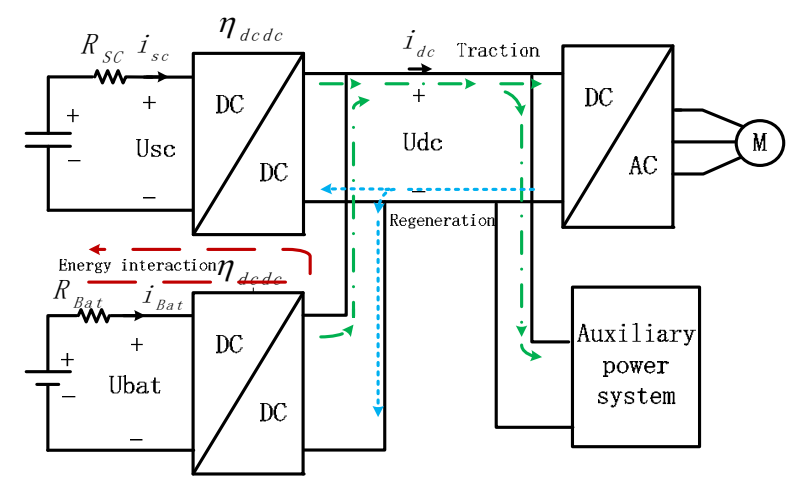

Figure 1: The topology of hybrid storage system. 
the charging and discharging current of the battery pack and supercapacitor pack should not exceed the current threshold.

Additionally, to ensure the quality of the storage system, the voltages and states of charge (SOC) were limited to a certain range, and the formula is expressed as follows:

$$
\left\{\begin{array}{l}
I_{b \min }<I_{b}(k)<I_{b \max } \\
I_{s c \min }<I_{s c}(k)<I_{s c \max } \\
S O C_{b \min }<S O C_{b s c}(k)<S O C_{b s c \max } \\
S O C_{s c \text { min }}<S O C_{s c}(k)<S O C_{s c \max } \\
k=1,2, \ldots, N
\end{array}\right.
$$

The EMS with a fixed power ratio is the method that the output ratio between battery pack and supercapacitor pack is fixed. When the required power is time varying according to real working condition, each pack will be asked to supply the charging or discharging power according to the ratio. The power equations of traction mode are as follows:

$$
\left\{\begin{array}{l}
P_{b}(k)=P_{r e q}(k) \cdot p_{b} / \eta_{d c} \\
P_{S C}(k)=P_{r e q}(k) \cdot\left(1-p_{s c}\right) / \eta_{d c} \\
p_{b}+p_{s c}=1
\end{array}\right.
$$

The power equations of regeneration mode are as follows:

$$
\left\{\begin{array}{l}
P_{b}(k)=P_{r e q}(k) \cdot p_{b} \bullet \eta_{d c} \\
P_{S C}(k)=P_{r e q}(k) \cdot\left(1-p_{s c}\right) \bullet \eta_{d c} \\
p_{b}+p_{s c}=1
\end{array}\right.
$$

where $\mathrm{p}_{\mathrm{b}}$ is the power ratio of battery pack, and $\mathrm{p}_{\mathrm{sc}}$ is the power ratio of the supercapacitor pack. The power distribution of this EMS is shown in Fig. 2. The ratio between the energy supplied by the supercapacitor pack (dark area) and the energy supplied by the battery pack (light area) of any part from Fig. 2 was fixed.

However, if the fixed power ratio method is put into use without improvement, in the case of the appearance of stations that consume too much energy, or the charging station breaks down or any other reason, the supercapacitor pack's SOC will be too low that it will influence the traction mode of the tram, and the hybrid storage system cannot work normally, thus,

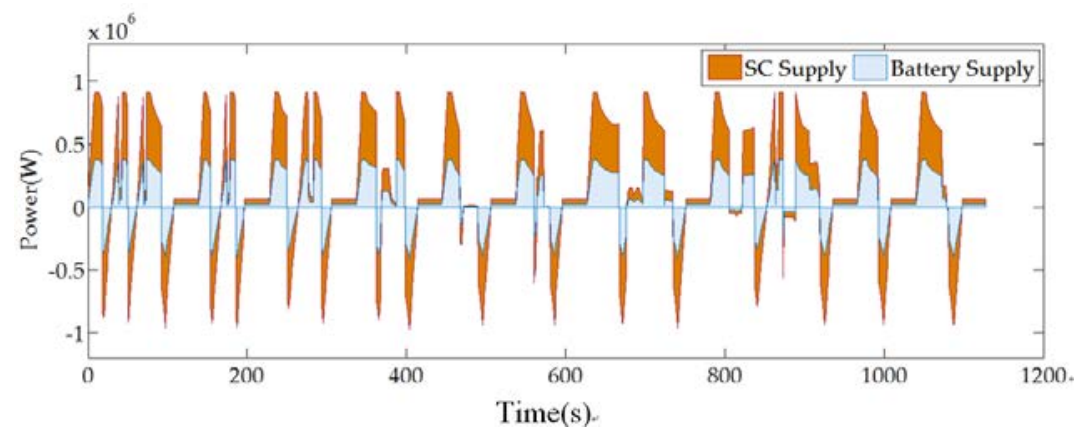

Figure 2: Power distributions of fixed power ratio strategy. 
causing deceleration or the tram to shut down. To prevent such conditions from happening, the EMS should be improved where the battery pack provides protection for the supercapacitor pack in special cases. At this time, the battery pack's output power will increase to the highest. If the power requirement of the tram is relatively low, the battery pack can provide whole traction power and charge for the supercapacitor pack at the same time, thus going into the energy interaction mode and restraining the supercapacitor's voltage from dropping off. The power equations for the special traction mode are shown as follows:

$$
\begin{aligned}
& P_{b}(k)= P_{b \max }(k) \\
& P_{s c}(k)=\left\{\begin{array}{r}
\left(P_{r e q}(k)-P_{b \max }(k) \bullet \eta_{d c}\right) \bullet \eta_{d c} \\
, P_{r e q}(k)>P_{b \max }(k) \\
\left(P_{r e q}(k)-P_{b \text { max }}(k) \bullet \eta_{d c}\right) / \eta_{d c} \\
, P_{r e q}(k)<P_{b \text { max }}(k)
\end{array}\right.
\end{aligned}
$$

If supercapacitor pack's $\mathrm{SOC}$ is lower than the protection threshold $\mathrm{SOC}_{\text {sclow }}$ when it is on a traction mode, the special mode will be launched, and when the SOC of the supercapacitor pack rises to another protection threshold $\mathrm{SOC}_{\text {schigh, }}$, the special mode will be quitted. This special mode can protect the supercapacitor from the problem of insufficient power, and shrink the working range, so it will help to reduce the sizing at the same time.

\section{SIZING OPTIMIZATION}

\subsection{Condition analysis}

Considering the real requirements of trams and lines, there are limitations during the optimization process.

The relationship of current, voltage, and power is defined as:

$$
i_{b}(k)=\frac{U_{b}(k)-\sqrt{U_{b}(k)^{2}-4 \cdot R_{b} \cdot P_{\mathrm{b}}(k)}}{2 \cdot R_{b}}
$$

where $U_{b}$ is the terminal voltage of the battery pack, and the limitation should be satisfied as eqn (11) shows:

$$
U_{b}(k)^{2}-4 \cdot R_{b} \cdot P_{\mathrm{b}}(k)>0
$$

where $\mathrm{U}_{\mathrm{sc}}$ is the terminal voltage of the supercapacitor pack.

The operation state of the storage system has changed on and on. To satisfy the power requirements at every moment, the real-time charging or discharging power of the battery pack $\left(\mathrm{P}_{\mathrm{b}}(\mathrm{t})\right)$ and the supercapacitor pack $\left(\mathrm{P}_{\mathrm{sc}}(\mathrm{t})\right)$ should satisfy the power requirements and bear the loss:

$$
P_{b}(k)+P_{s c}(k) \geq P_{r e q}(k)+P_{l o s}(k)
$$

There are some charging stations during the line, which supplements the storage system. As a result, the whole energy requirement of the tram can be supplied by the initial energy that the storage system brings and the charging energy that charging stations provide, as shown in eqn (13): 


$$
E_{b}+E_{S C}+E_{c h a r} \geq E_{\text {req }}+E_{\text {los }}
$$

where $E_{b}, E_{s c}, E_{c h a r}, E_{\text {req }}$, and $E_{\text {los }}$ are the initial energy of battery and supercapacitor, the whole energy supplied in charging stations, the whole energy requirement of tram and the whole energy loss, respectively.

If the hybrid storage system is installed on the tram, it should not be too heavy to limit the normal operation and number of passengers. The limitation is shown in the following equation:

$$
M_{b}+M_{S C} \leq M_{\lim }
$$

where $\mathrm{M}_{\mathrm{b}}$, and $\mathrm{M}_{\mathrm{sc}}$ are the weight of the battery pack and the supercapacitor pack, respectively, and $\mathrm{M}_{\mathrm{lim}}$ is the maximum weight limitation of the hybrid storage system.

Taking cost and maintenance into consideration, the change times should not be too frequent. Therefore, the battery lifespan is required to be long enough. The criterion directly influences the ratio of the battery's output energy. At the same time, the appropriate working range of SOC can also prolong the lifespan of the storage system:

$$
\begin{aligned}
& S O C_{b \min } \leq S O C_{\mathrm{b}}(k) \leq S O C_{b \max } \\
& S O C_{s c \min } \leq S O C_{\mathrm{sc}}(k) \leq S O C_{s c \max }
\end{aligned}
$$

\subsection{Optimization process}

Sizing optimization makes interaction effective with EMS [12], [13]. A good EMS can improve the utilization of an energy storage system, and can reduce the sizing based on the system requirements. In order to verify the superiority of the EMS with energy interaction, the objective of the optimization is the weight of hybrid storage system.

The variables of optimization are series numbers and parallel numbers of battery and supercapacitor. After determining the parameters of a set of optimized variables, the energy, power and other parameters of the energy storage system have been determined.

The optimization formula of sizing is as follows:

$$
\begin{aligned}
& \min F(\vec{N})=m_{b} \bullet N_{b s} \bullet N_{b p}+m_{s c} \cdot N_{s c s} \bullet N_{s c p} \\
& \vec{N}=N_{b s}, N_{b p}, N_{s c s}, N_{s c p}
\end{aligned}
$$

where $\mathrm{N}_{\mathrm{bs}}, \mathrm{N}_{\mathrm{bp}}, \mathrm{N}_{\mathrm{scs}}$, and $\mathrm{N}_{\mathrm{scp}}$ are the series and parallel numbers of the battery pack and the supercapacitor pack, respectively; and $\mathrm{mb}$, msc are the weights of the battery cell and supercapacitor cell.

In this paper, the optimal value of the established target was obtained by optimizing the series and parallel numbers of the battery pack and supercapacitor pack, which was suitable for solving the problem by using the intelligent optimization algorithm. This paper used an improved particle swarm optimization algorithm. The steps of sizing optimization are shown in Fig. 3.

The software is divided into two parts. The main program is to select and optimize the variables in the selected value range using the optimization algorithm. Each group of variables on behalf of a certain hybrid storage system generated by the main program will enter the subprogram to perform the real-time operation simulation under the required EMS; the ones that meet the constraints are reserved and go into the next iteration, and the others are eliminated with the assignment of the minimum fitness value. 


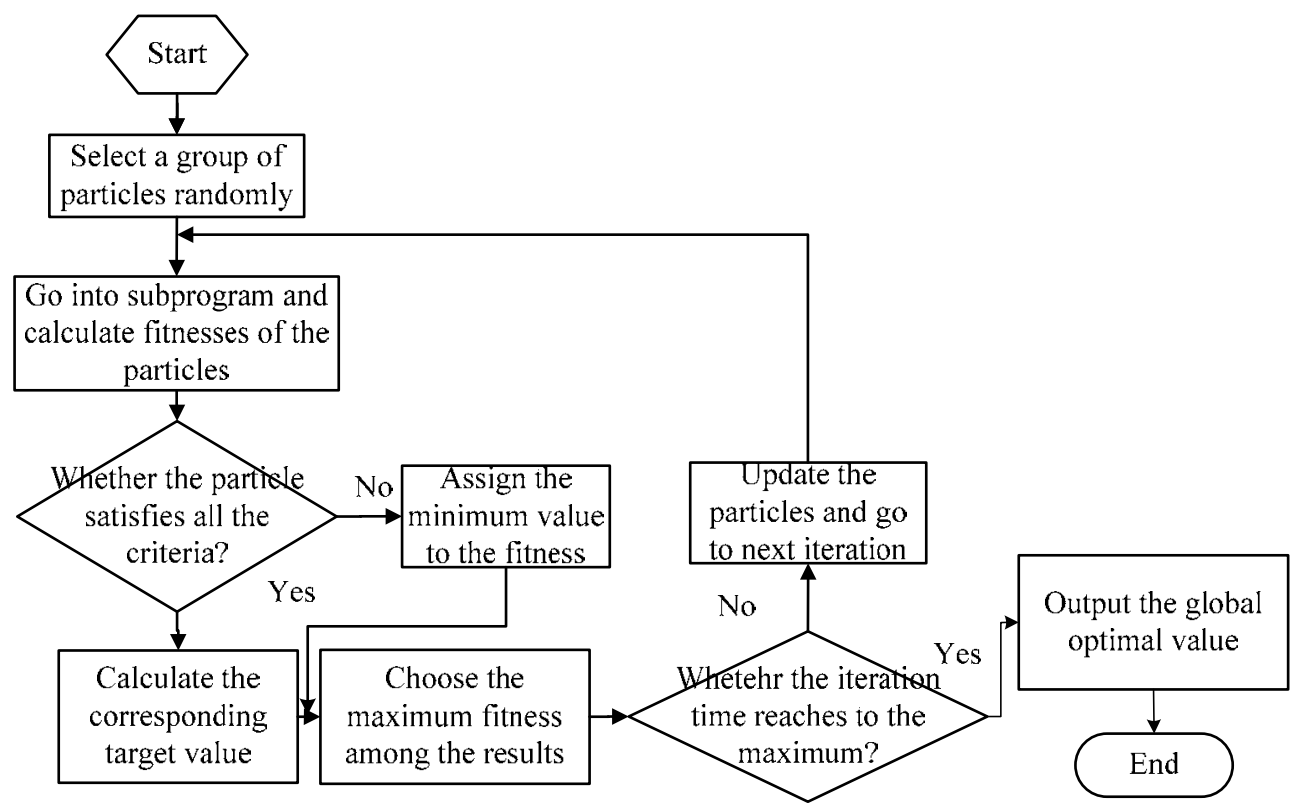

Figure 3: Flow diagram of sizing optimization.

\section{SIMULATION ANALYSIS AND EXPERIMENT VALIDATION}

\subsection{Simulation analysis}

The paper selected a defined tram line as the study object, where the tram drives 20 cycles every day. The main parameters of the tram and line are shown in Table 1. Every charging station provides 20 seconds' charging time; during this time, the charging post offers $1500 \mathrm{~A}$ charging current to the DC bus, and the charging power ratio is the same with operation.

On the basis of the above criteria, the power curve was obtained by traction simulation, as shown in Fig. 4.

The important input parameters of collaborative optimization of EMS and sizing are shown in Table 2.

To prove the effect of sizing caused by energy interaction state, the paper makes sizing optimizations based on the EMS with and without energy interaction, respectively.

Table 1: Parameters of tram and line.

\begin{tabular}{|l|c|c|c|}
\hline Parameter & Value & Parameter & Value \\
\hline Formation & 2M3T & Stop station & 11 \\
\hline Weight $(\mathrm{t})$ & $75(\mathrm{AW} 3)$ & Charging station & 7 \\
\hline Bus voltage $(\mathrm{V})$ & DC750 & $\begin{array}{c}\text { Maximum velocity } \\
(\mathrm{km} / \mathrm{h})\end{array}$ & 70 \\
\hline Line length $(\mathrm{km})$ & 9.44 & $\begin{array}{c}\text { Average velocity } \\
(\mathrm{km} / \mathrm{h})\end{array}$ & 28 \\
\hline
\end{tabular}




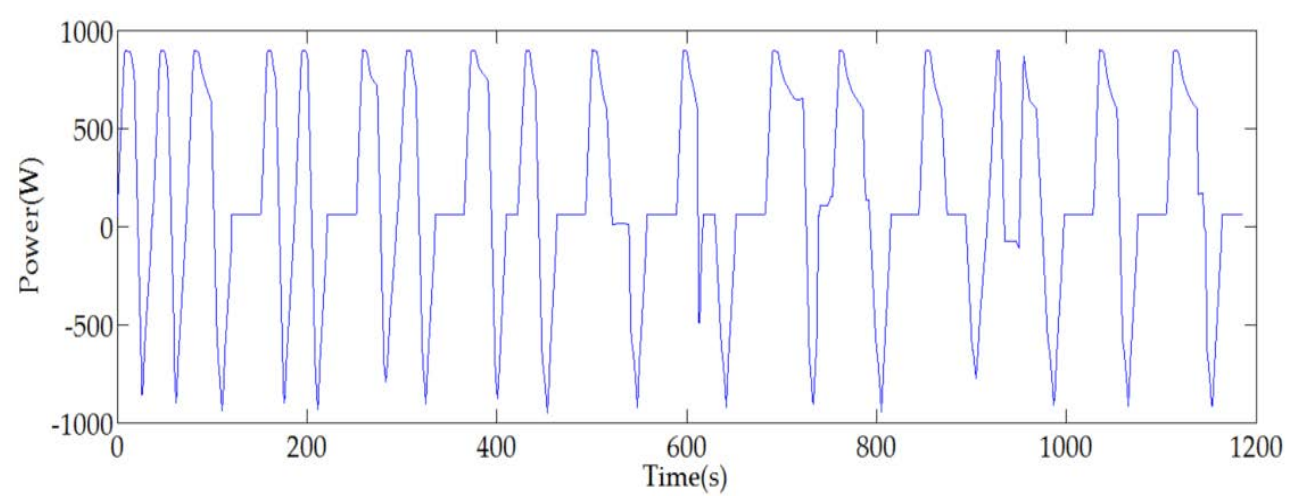

Figure 4: Power curve.

Table 2: Important input parameters.

\begin{tabular}{|l|c|c|c|}
\hline Parameter & Value & Parameter & Value \\
\hline $\mathrm{p}_{\mathrm{b}}$ & 0.3 & $\eta_{d c}$ & $95 \%$ \\
\hline $\mathrm{SOC}_{\mathrm{bmax}}$ & $80 \%$ & $\mathrm{SOC}_{\text {scmax }}$ & $100 \%$ \\
\hline $\mathrm{SOC}_{\mathrm{bmin}}$ & $30 \%$ & $\mathrm{SOC}_{\text {scmin }}$ & $25 \%$ \\
\hline $\begin{array}{l}\text { Maximum } \\
\text { iterations }\end{array}$ & 30 & Size of particle swarm & 500 \\
\hline SOC $_{\text {sclow }}$ & 0.4 & SOC $_{\text {schigh }}$ & 0.7 \\
\hline
\end{tabular}

The types of battery and supercapacitor have large influence on sizing result, for the energy density and power density directly decide how many cells the system allocates, the higher the energy density and power density are, the lighter the storage system is. Lithium titanate battery has the advantages of high power density and relatively long lifespan among types of batteries, so it is popular among high power applications. After wide investigation about characteristics and price, the paper finally chooses MV06203127NTP from Microvast. Maxwell has been studying and manufacturing supercapacitor for many years and the quality can be guaranteed. The paper chooses BCAP3000 from Maxwell with both long lifespan and good characteristics. The main parameters of battery and supercapacitor are shown in Table 3 and the sizing results are shown in Table 4.

As we can see from the total weight, the optimized sizing under the EMS with no energy interaction was 2.27 tons heavier than that with energy interaction, but the total energy has no big change, it is due to the difference of supercapacitor configuration. Because of the low energy density, the more the supercapacitor configures, the heavier the system is. Under energy interaction state, the battery can provide energy for the supercapacitor. Under the same configuration, it can reduce the working scope of the supercapacitor, and then reduce the configuration. There is an increase on battery configuration, it is due to the accessorial output of battery, for the battery should not only provide traction power and also replenish 
Table 3: Parameters of battery and supercapacitor.

\begin{tabular}{|c|c|c|}
\hline Parameter & MV06203127NTP & BCAP3000 \\
\hline Rated voltage $(\mathrm{V})$ & 2.3 & 2.7 \\
\hline Rated capacity & $10 \mathrm{Ah}$ & $3000 \mathrm{~F}$ \\
\hline Internal resistance $(\mathrm{m} \Omega)$ & $1.4\left(\mathrm{R}_{\mathrm{b}}\right)$ & $0.29\left(\mathrm{R}_{\mathrm{sc}}\right)$ \\
\hline Maximum continuous current $(\mathrm{A})$ & $30(3 \mathrm{C})$ & 200 \\
\hline Energy $(\mathrm{Wh})$ & 23 & 3.04 \\
\hline Weight $(\mathrm{kg})$ & 0.3 & 0.51 \\
\hline Cycle life & 10,000 & $2,000,000$ \\
\hline
\end{tabular}

Table 4: Sizing optimization results.

\begin{tabular}{|l|c|c|}
\hline Parameter & $\begin{array}{c}\text { Without energy } \\
\text { interaction }\end{array}$ & $\begin{array}{c}\text { With energy } \\
\text { interaction }\end{array}$ \\
\hline Battery cell numbers & 2002 & 3090 \\
\hline Supercapacitor cell numbers & 7855 & 2790 \\
\hline Battery's energy(kWh) & 46 & 71 \\
\hline Supercapacitor's energy $(\mathrm{kWh})$ & 23.9 & 8.48 \\
\hline Total weight $(\mathrm{t})$ & 4.6 & 2.33 \\
\hline
\end{tabular}

energy for supercapacitor. However, battery has high energy density, so the battery pack has little increase in weight.

To conclude, the overmuch configuration of a supercapacitor for the common method brought about the overweight of the hybrid storage system, and also increased the volume and initial cost of the system. Conversely, the weight, volume, and initial cost of the optimized sizing for the strategy of energy interaction were effectively reduced. This is meaningful for the onboard storage system, and the tram will have a larger space for passengers.

\subsection{Experiment validation}

To verify the superiority of the EMS with energy interaction, a contrast experiment between the common method and the special method was undertaken using the $90 \mathrm{~kW}$ batterysupercapacitor hybrid storage platform. Photo of the complete experiment platform is shown as Fig. 5. The main parameters of the platform are shown in Table 5.

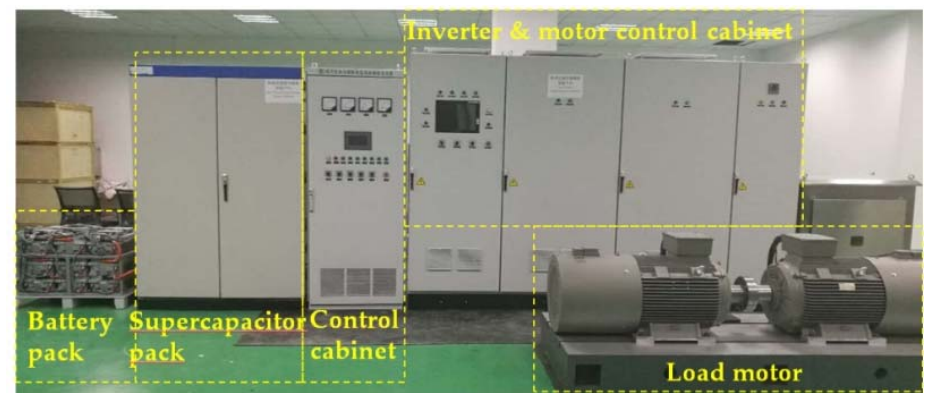

Figure 5: Photo of platform. 
Table 5: Parameters of hybrid storage platform.

\begin{tabular}{|l|c|c|}
\hline Parameter & Battery pack & $\begin{array}{c}\text { Supercapacitor } \\
\text { pack }\end{array}$ \\
\hline Type & MV0620312 & BCAP3000 \\
\hline Series and parallel numbers & $216 \mathrm{~S} 2 \mathrm{P}$ & $184 \mathrm{~S} 1 \mathrm{P}$ \\
\hline Total energy (kWh) & 14 & 0.5 \\
\hline Maximum continuous current (A) & $60(3 \mathrm{C})$ & 200 \\
\hline Terminal voltage $(\mathrm{V})$ & 496.5 & 500 \\
\hline
\end{tabular}

The block diagrams of the common state's and energy interaction state's control strategies are shown in Fig. 6(a) and (b). Under the EMS with a fixed power ratio, the control strategy used the DC bus voltage's outer loop and the inner loops of the battery and supercapacitor packs to make double loop controls. The product of the supercapacitor voltage and the output of the voltage outer loop was required power, and its ratio distributions were inputted into the inner loops to act on the battery and supercapacitor packs.

However, the block diagram of the energy interaction state in the special method was different from the above one, which is shown in Fig. 6(b). The battery pack is mounted to a power source that supplies constant current output, and battery is controlled by a single current loop in this case. As for the supercapacitor pack, it is the only load and should absorb all the power supplied by the battery pack and motor, so the control loops of the supercapacitor pack are changeless when compared with Fig. 6.

The power curve from Fig. 4 made a 60 -fold reduction to be the input curve of the load motor. The contrast experiment chose the curve of the first 180 seconds, and the initial voltages of supercapacitor pack were both around $395 \mathrm{~V}$. To make the EMS more obvious, the power ratio of battery pack was set to 0.3 and the $\mathrm{SOC}_{\text {sclow }}$ and $\mathrm{SOC}_{\text {schigh }}$ were 0.4 and 0.7 , respectively. The battery pack's output current was $35 \mathrm{~A}$ at the special state. The supercapacitor's SOC curves as well as the current curves of the battery and supercapacitor packs are shown in Figs 7 and 8, respectively.

The two curves (a) and (b) from Fig. 7 are the supercapacitor's SOC curves under the special and common EMS, respectively. It should be pointed out that the battery pack had a quantity of energy when compared with the energy used in this experiment, thus its voltage had a slight fluctuation, so we only paid attention to the states of the supercapacitor pack. Figs 8(a) and 8(b) show the comparison of the current curves of the battery and supercapacitor packs between the different energy management strategies. It was easy to notice that the special state was activated when the supercapacitor pack's SOC was lower than $\mathrm{SOC}_{\text {sclow }}$, and the battery pack started to discharge with the constant current of $35 \mathrm{~A}$, as seen from Figure $8 \mathrm{a}$. The required power was lower than the battery pack's output power, thus the excessive power will charge the supercapacitor pack, and the supercapacitor pack's SOC continuously rose until it obtained the $\mathrm{SOC}_{\text {schigh }}$. As shown in Fig. 8(a), the battery pack recovered to the common state, that is to say that it will no longer discharge at a high current, and the hybrid storage system comes back to the working state that distributes the output power according to the power ratio. After energy supplement in the special state, the supercapacitor pack's SOC was relatively high. However, according to the curves with the common EMS shown in Fig. 8(b), the battery and supercapacitor packs kept the power ratio of 3:7, and there was no energy interaction when the supercapacitor pack's SOC was low. After four traction modes, the supercapacitor pack's SOC fell to 0.3 , so it could not supply 


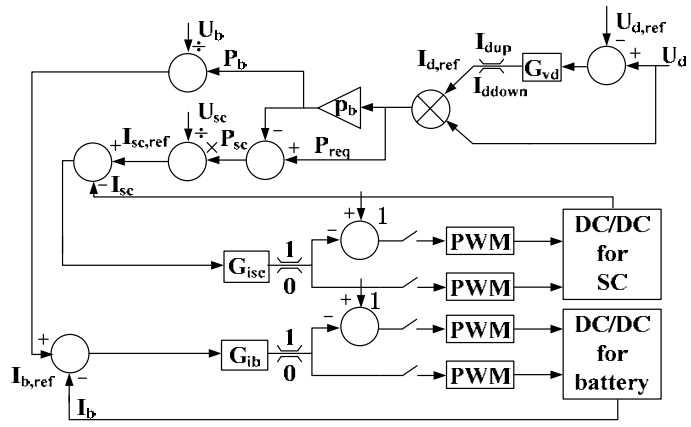

(a)

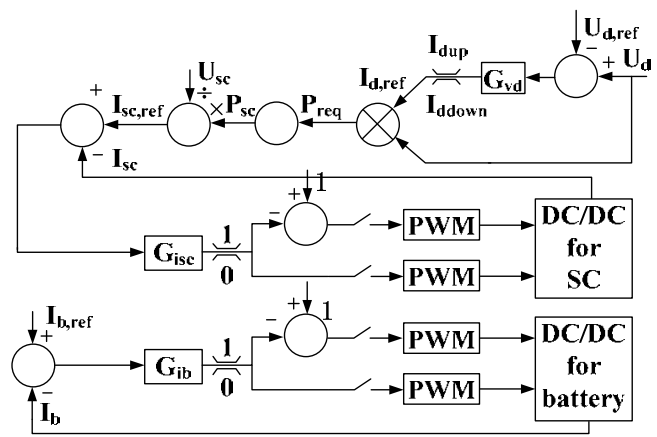

(b)

Figure 6: Control strategies of the system.

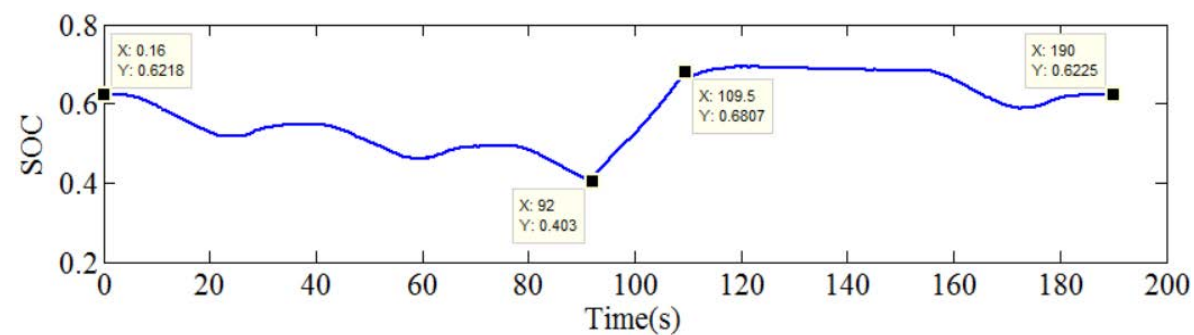

(a)

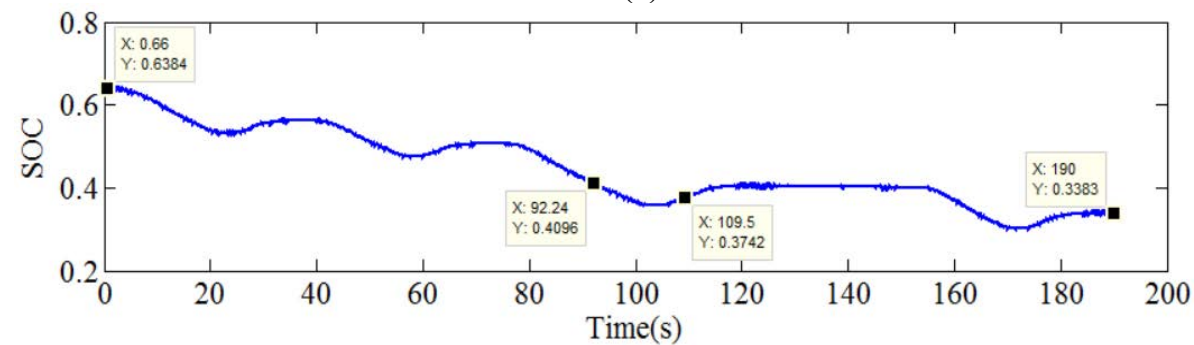

(b)

Figure 7: The supercapacitor pack's SOC curves. 


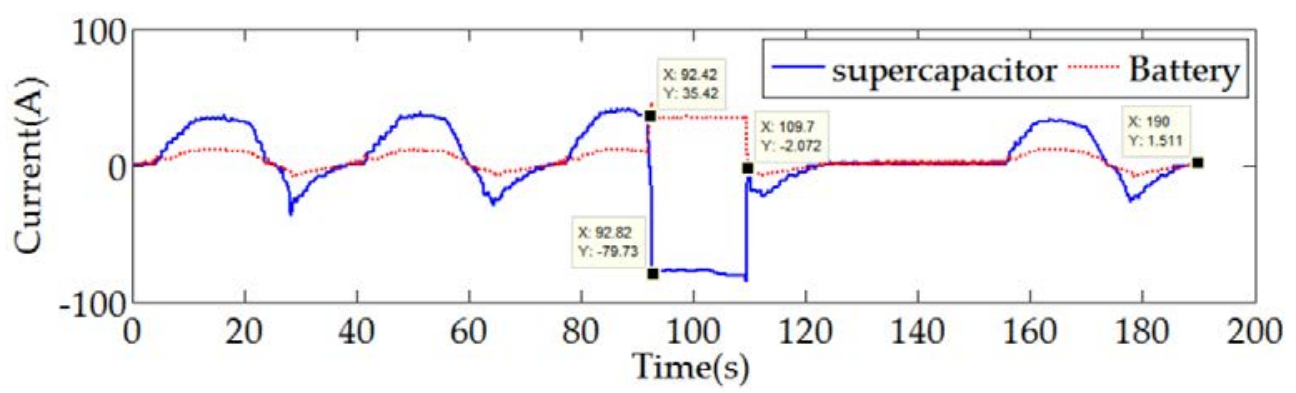

(a)

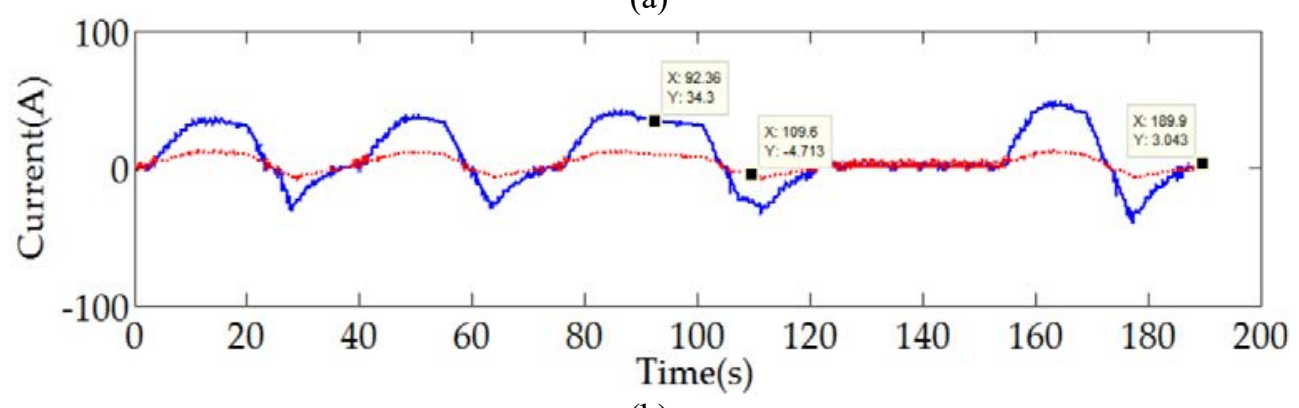

(b)

Figure 8: The current curves of the battery and supercapacitor packs.

power normally at the next traction mode. The voltage difference at the end was about 100 $\mathrm{V}$ between the two methods, and the difference increased with time.

As shown by the experiment data, the special state provides a timely supplement for the supercapacitor pack's energy. In contrast, the EMS with a fixed power ratio has made a continuous decline on the SOC of the supercapacitor pack and cannot continue normal operation if there is no energy supply at the stop station. Therefore, the hybrid storage system cannot provide power for the whole journey of the tram. To maintain normal operation, the sizing of supercapacitor will be enlarged.

Compared with the experiment results based on the strategy without energy interaction (which are shown in Figs 7(b) and 8(b)), the improved strategy guaranteed the dynamic equilibrium of SOC of the supercapacitor pack, thus offering advantages in engineering.

\section{CONCLUSIONS}

In this paper, a fixed power ratio distribution strategy with energy interaction was put forward for the tram's hybrid storage system, and the sizing was optimized, and the superiority of EMS was verified through experiment. This paper analyzed the online EMS and made improvement, adding energy interaction between battery and supercapacitor, and made collaborative sizing optimization based on line conditions. The sizing result verifies the preponderance of the improved EMS, which helped to reduce weight and cost of hybrid storage system. And the contrast experiment between the EMS with and without energy interaction state made a step further to verify the superiority of the improved EMS.

The experiment platform can verify all kinds of online EMSs, however, the efficiency is low, so the next step is to analysis the hardware and to improve the efficiency. 


\section{REFERENCES}

[1] Wang, B., Power-split hybrid electric vehicle energy management based on improved logic threshold approach. Mathematical Problems in Engineering, pp. 1-9, 2013.

[2] Zhao, G.Y. et al., Energy management strategy for series hybrid electric vehicle. Journal of Northeastern University, 34(4), pp. 583-587, 2013.

[3] Huang, Y.J., Yin, C.L. \& Zhang, J.W., Design of an energy management strategy for parallel hybrid electric vehicles using a logic threshold and instantaneous optimization method. International Journal of Automotive Technology, 10(4), pp. 513-521, 2009.

[4] Akli, C.R. et al., Energy management and sizing of a hybrid locomotive. European Conference on Power Electronics and Applications IEEE, pp. 1-10, 2008.

[5] Hredzak, B., Agelidis, V.G. \& Demetriades, G., A Low Complexity Control System for a Hybrid Battery-Ultracapacitor Power Source, 2013.

[6] Motapon, S.N., Dessaint, L.A. \& Al-Haddad, K., A comparative study of energy management schemes for a fuel-cell hybrid emergency power system of more-electric aircraft. IEEE Transactions on Industrial Electronics, 61(3), pp. 1320-1334, 2013.

[7] He, H. et al., Energy management strategy research on a hybrid power system by hardware-in-loop experiments. Applied Energy, 112(16), pp. 1311-1317, 2013.

[8] Patil, R.M., Filipi, Z. \& Fathy, H.K., Comparison of supervisory control strategies for series plug-in hybrid electric vehicle powertrains through dynamic programming. IEEE Transactions on Control Systems Technology, 22(2), pp. 502-509, 2014.

[9] Wang, X. \& Liang, Q., Bidirectional energy management for plug-in hybrid electric vehicles via vehicle-to-grid. Communications, Signal Processing, and Systems, Springer: New York, pp. 4197-4201, 2012.

[10] Johnson, V.H., Battery performance models in ADVISOR. Journal of Power Sources, 110(2), pp. 321-329, 2002.

[11] Guzzella, L. \& Sciarretta, A., Vehicle propulsion systems. Introduction to Modeling and Optimization, 26(1), pp. 67-162, 2007. 